\title{
CARACTERÍSTICAS DO SÊMEN DE TAMBAQUI (COLOSSOMA MACROPOMUM) EM LATITUDE EQUATORIAL\#
}

\author{
TAMBAQUI (COLOSSOMA MACROPOMUM) SEMEN CHARACTERISTICS \\ ONEQUATORIAL LATITUDE
}

\author{
Vieira, M.J.A.F. ${ }^{*}$, Carvalho, M.A.M. ${ }^{1}$, Salmito-Vanderley, C.S.B. ${ }^{1}$, Salgueiro, C.C. de M. ${ }^{1}$, \\ Viveiros, A.T.M. ${ }^{2}$, Moura, A.A.A.N. ${ }^{1}$ e Nunes, J.F. ${ }^{1}$
}

${ }^{1}$ Universidade Estadual do Ceará (UECE). Fortaleza. Brasil. *ascensaof@bol.com.br

${ }^{2}$ Universidade Federal de Lavras. Minas Gerais. Brasil.

\section{PalaVRas CHAVE ADICIONAIS}

Nordeste do Brasil. Reprodução. Peixes. Teleósteos.

\section{RESUMO}

Otambaqui Colossoma macropomum (Cuvier, 1818) é uma espécie de teleósteo da bacia amazônica e abundante naquela região, de alto valor comercial e forte apelo culinário e esportivo sendo uma das principais espécies amazônicas com larga distribuição nos rios e nas áreas próximas a Manaus (Silva et al., 1984) Este trabalho teve por objetivo avaliar as características espermáticas do sêmen de tambaqui com e sem indução hormonal por um período de doze meses. Utilizou-se 26 machos com idade média de três anos, pertencentes ao Centro de Pesquisas em Aquicultura (CPAq) do Departamento Nacional de Obras Contra às Secas (DNOCS), devidamente identificados com chips magnéticos. Os animais foram divididos em dois grupos: induzidos (I) e não induzidos ( $\mathrm{NI}$ ). Os animais do grupo I receberam 2 $\mathrm{mg}$ de extrato de pituitária de carpa $\left(\mathrm{EPC}^{-1}\right)$ por $\mathrm{kg}$ de peso vivo (PV), via intra celomática. O sêmen foi coletado após 14 horas através de massagem abdominal. Os parâmetros avaliados foram: volume, $\mathrm{pH}$, osmolaridade e concentração espermática. Os peixes apresentaram peso médio de $5410 \mathrm{~g}$, comprimento total de $68 \mathrm{~cm}$ e comprimento padrão de $59,17 \mathrm{~cm}$. Para os grupos I e NI foram observados respectivamente: volume médio de $5,05 \mathrm{~mL} e$

"Experimento de tese de Doutorado do primeiro autor junto ao Programa de Pós-Graduação em Ciências Veterinárias da Universidade Estadual do Ceará.

Recibido: 21-5-09. Aceptado: 12-5-10.

\section{AdDitional KeYWORDS}

Northeast of Brazil. Reproduction. Fish. Teleostei.

$0,55 \mathrm{~mL}(\mathrm{p}<0,05) ; \mathrm{pH}$ médio de 8,21 e 8,09; osmolaridade média de 320,51 e $323 \mathrm{mOsm} / \mathrm{kg} \mathrm{e}$ concentração espermática média de 22,93 × $10^{9}$ e $40,46 \times 10^{9} \mathrm{sptz} / \mathrm{mL}(p<0,05)$. A produção espermática total foi de $115,79 \times 10^{9} \mathrm{sptz} / \mathrm{mL}$ no grupo I sendo de $22,25 \times 10^{9} \mathrm{sptz} / \mathrm{mL}$ no grupo NI. Não houve diferença significativa entre os valores obtidos de osmolaridade e $\mathrm{pH}$ dos indivíduos induzidos e não induzidos. No entanto, a concentração espermática e o volume no grupo induzido apresentaram valores significativamente superiores $(p<0,05)$ comparado aos indivíduos não induzidos.

\section{SUMMARY}

The Amazonian fish known as tambaqui (Colossoma macropomum Cuvier, 1818) is a osseous fish and has high commercial value because of its meat quality and for recreational fishing. Tambaqui is one of the major species in the Amazon basin and found in most rivers and surroundings of Manaus city (Silva et al., 1984). The present study was carried out to evaluate sperm parameters from tambaqui, subjected or not to hormone treatment for a period of 12 months. We used 26 males with three years of age, raised at the Aquaculture Research Center of the National Department for Drought Control (DNOCS). Animals were identified with magnetic chips and divided into two groups: controls (NI) and hormone-treated 
(I), which received $2 \mathrm{mg}$ of carp pituitary extract $\left(\mathrm{CPEkg}^{-1}\right)$ of body weight (via intra celomic). Semen was collected 14 hours after hormonal induction and the following parameters evaluated: volume, $\mathrm{pH}$, osmolarity and sperm concentration. Fishes had body weight of $5410 \mathrm{~g}$, with total length and standard length of $68 \mathrm{~cm}$ and $59.17 \mathrm{~cm}$, respectively. In the case of hormone-treated and control animals, we found the following criteria, respectively: semen volume of $5.05 \mathrm{~mL}$ and 0.55 $\mathrm{mL}(\mathrm{p}<0.05) ; \mathrm{pH}$ of 8.21 and 8.09 ; osmolarity of 320.51 and $323 \mathrm{mOsm} / \mathrm{kg}$; sperm concentration of $22.93 \times 10^{9}$ and $40.46 \times 10^{9} \mathrm{sptz} / \mathrm{mL}(p<0,05)$. Total sperm productions were quantified as $115.79 \times$ $10^{9} \mathrm{sperm} / \mathrm{mL}$ in treated fishes and $22.25 \times 10^{9}$ sperm $/ \mathrm{mL}$ in the controls. We conclude that treatment of adult tambaqui with carp pituitary extract increase semen volume but reduces sperm concentration while had no effect on sperm osmolarity and $\mathrm{pH}$.

\section{INTRODUÇÃO}

O tambaqui Colossoma macropomum, Cuvier (1818), Characidae, Characiforme, originário da bacia amazônica, encontra-se amplamente distribuído por seus rios e afluentes e em áreas próximas à Manaus. Em 1972, o Departamento Nacional de Obras Contra às Secas (DNOCS) introduziu no Nordeste do Brasil 74 alevinos oriundos de Iquitos, Peru, que deram início à colossomicultura no Ceará (Silva et al., 1984). A obtenção da primeira desova artificial foi em 1977, sendo então os alevinos distribuídos para todo o Nordeste. O período reprodutivo desta espécie em ambiente nativo vai de setembro a fevereiro com desovas ocorrendo entre os meses de setembro/outubro até janeiro/fevereiro (Villacorta-Correa e SaintPaul, 1999). Durante este período alimentase de frutos e sementes como fonte de energia para o desenvolvimento e a maturação gonadal, (Silva et al., 1999). Em cativeiro, sob estresse de confinamento, o tambaqui perde a capacidade de se reproduzir naturalmente, sendo necessária a indução à desova através da aplicação de hormônios gonadotróficos exógenos. Diversos estudos têm avaliado o efeito de indutores hormonais sobre a capacidade reprodutiva desta espécie (Silva et al., 1981; Ponzi Junior, 2003; Muniz, 2006).

Aspectos seminais têm sido divulgados para algumas espécies de peixes, no entanto, quando se faz referência a efeitos da sazonalidade nas espécies reofílicas de águas tropicais sobre a qualidade espermática estes dados são bastante escassos (Kavamoto et al., 1986; Shimoda et al., 1999; Andrade-Talmelli et al., 2001; Shimoda, 2004; Cruz-Casallas et al., 2005) não tendo evidência de estudos que avaliem a qualidade seminal do tambaqui em cativeiro e sua variação anual.

A qualidade do sêmen é fundamental para o processo reprodutivo, pois a contribuição do macho para a eficiência reprodutiva e produtiva é de grande importância, uma vez que além do aporte genético, neles pode se aplicar uma maior e mais rápida pressão na seleção. Fatores como a concentração espermática, aspecto, e volume do ejaculado, são de grande significância para o bom desempenho reprodutivo aliado às qualidades espermáticas como velocidade progressiva e percentual de células vivas (Salgueiro e Nunes, 1999). Portanto, este trabalho teve por objetivo avaliar as características espermáticas do sêmen do tambaqui com e sem indução hormonal por um período de doze meses.

\section{MATERIALE MÉTODOS}

O experimento foi conduzido no Centro de Pesquisas em Aquicultura (CPAq) do DNOCS, no município de Pentecoste, Ceará, situando-se a $3^{\circ} 45^{\prime} 00^{\prime \prime}$ de latitude sul e $39^{\circ} 10^{\prime} 24^{\prime \prime}$ de longitude oeste, distante $82 \mathrm{~km}$ da Capital Fortaleza, e no Núcleo Integrado de Biotecnologia (NIB) da Universidade Estadual do Ceará (UECE) em Fortaleza, Ceará, Brasil, no período entre 2007 e 2008. Os animais foram mantidos em viveiros de terra de $350 \mathrm{~m}^{2}$ abastecidos pela água do açude Pereira de Miranda. A temperatura 
ambiental média foi de $26,8^{\circ} \mathrm{C}$, com máxima de $34^{\circ} \mathrm{C}$ e mínima de $20,6^{\circ} \mathrm{C}$. O período de chuvas estendeu-se de janeiro a junho e a pluviosidade média anual foi de $860 \mathrm{~mm}$ (FUNCEME, 2008).

Foram utilizados 26 machos da espécie tambaqui (Colossoma macropomum, Cuvier, 1818), devidamente identificados através de chips, para evitar perdas dos dados, e agrupados em pares $(n=13)$ e impares $(n=$ 13), de acordo com o número do chip. Os animais aptos à reprodução com idade média superior a três anos foram selecionados, sendo em seguida pesados (g) e medidos (cm). Receberam ração comercial contendo $32 \%$ de proteína bruta com base em $5 \%$ do peso vivo (PV) dividida em duas refeições diárias, as oito e às 16 horas.

Os animais selecionados receberam dose única em cada aplicação de extrato pituitário de carpa comum (EPC) (Cyprinus carpio, 2 $\mathrm{mg} / \mathrm{kg} \mathrm{PV}$ ), por via intra celomática, na base da nadadeira peitoral. Os animais foram submetidos a um regime de rodízio de maneira que em cada mês um grupo tinha o sêmen coletado após indução com EPC (I) enquanto que o outro grupo tinha o sêmen coletado sem indução (NI). Deste modo, todos os animais recebiam EPC a cada 60 dias. Os animais impares foram induzidos nos meses de abril, junho, agosto, outubro e dezembro de 2007 e fevereiro e abril de 2008, já os animais pares foram induzidos nos meses de maio, julho, setembro e novembro de 2007 e janeiro e março de 2008. Os dados médios do grupo I são referentes aos animais com numeração par e ímpar, da mesma forma que o grupo NI.

Durante a coleta os animais foram submetidos à anestesia com solução a base de óleo de cravo (União Vegetal Suplementos Nutricionais Ltda), usado como se segue: $1 \mathrm{~mL}$ do óleo de cravo diluído em 10 $\mathrm{mL}$ de álcool absoluto e $1 \mathrm{~mL}$ desta solução mãe foi diluída em $1000 \mathrm{~mL}$ de água obtendo uma diluição final 1:10000. Cada animal foi individualmente mergulhado no tanque para anestesia com capacidade de 70 litros, contendo a solução anestésica por aproximadamente 2 minutos, até que fosse detectado o sintoma principal da perda de equilíbrio (ventre voltado para cima). Atingindo este estado, o peixe era imediatamente retirado do tanque e submetido à coleta de sêmen, mensuração do comprimento e pesagem. Após estes procedimentos o mesmo era devolvido ao tanque de manuseio para se recuperar totalmente da anestesia.

As coletas de sêmen foram realizadas após 14 horas da indução hormonal. Cada animal foi contido em decúbito dorsal e envolto em pano úmido sobre os olhos para facilitar contenção. O orifício genital foi enxuto com papel toalha, e uma pressão abdominal foi realizada no sentido anteroposterior conforme método adotado rotineiramente pelo DNOCS. O sêmen liberado em alíquotas foi coletado em tubos de polipropileno de 1,5 $\mathrm{mL}$ graduados. Logo após, foi aferido o volume (mL) e o $\mathrm{pH}(0-14$; mediante uso de fitas reagentes). Alíquotas de $1 \mathrm{~mL}$ foram retiradas e diluídas em $4000 \mathrm{~mL}$ de solução salina formolizada a $1 \%$ para contagem de espermatozóides em Câmara de Neubauer e $100 \mathrm{~mL}$ para leitura da osmolaridade $(\mathrm{mOsm} / \mathrm{kg})$ (Osmometer Automatic, Roebling, Alemanha). Para avaliar o percentual de espermatozoides móveis, uma alíquota de sêmen de $2 \mu \mathrm{L}$ foi diluída em $50 \mu \mathrm{L}$ de água do açude e uma estimativa subjetiva da motilidade foi realizada em microscópio ótico em aumento de $100 \mathrm{x}$, considerando-se como 0 (zero) nenhum espermatozoide móvel e 100 (cem) todos espermatozoides móveis. As amostras foram identificadas e conservadas em isopor com gelo em escamas a uma temperatura de $\pm 5^{\circ} \mathrm{C}$ para o transporte até o Laboratório do Núcleo Integrado de Biotecnologia da UECE.

Os dados foram apresentados como média \pm desvio padrão. As médias apresentadas com intervalo de confiança de $95 \%$ foram testadas através de análise de variância em duas vias para verificar a 
diferença entre os animais dos grupos induzido e não induzido. O teste de Tukey foi utilizado para comparar as médias entre os tratamentos $(\mathrm{p}>0,05)$.

\section{RESULTADOSEDISCUSSÃO}

Os valores médios para os parâmetros de volume, $\mathrm{pH}$, concentração espermática e osmolaridade dos grupos induzidos (I) e não induzidos (NI) encontram-se na tabela I. Para melhor compreensão dos dados, os animais quando induzidos com EPC foram referenciados como grupo (I) enquanto que, os animais quando não sofreram indução foram referenciados (NI). Os animais do grupo I apresentaram um peso médio de $5410 \pm 289 \mathrm{~g}$, comprimento total de $68 \pm 1,8$ cm e comprimento padrão de $59,17 \pm 1,80 \mathrm{~cm}$, enquanto no grupo NI apresentaram peso médio de $5452,00 \pm 339 \mathrm{~g}$, comprimento total de $68,00 \pm 2,39 \mathrm{~cm}$ e comprimento padrão de $59,00 \pm 2,03 \mathrm{~cm}$. Foi observada concentração espermática média de $22,66 \pm 9,7 \times 10^{9} \mathrm{sptz} /$ $\mathrm{mL}$ para o grupo I e $40,46 \pm 18,13 \mathrm{sptz} / \mathrm{mL}$ para o grupo NI, como mostra a tabela I. A menor concentração espermática registrada para o grupo I foi de $9,12 \times 10^{9} \mathrm{sptz} / \mathrm{mL}$ no mês de janeiro de 2008 , enquanto a maior foi $44,04 \times 10^{9} \mathrm{sptz} / \mathrm{mL}$ no mês de maio de 2007 (figura 1). O grupo NI diferiu significativamente $(p<0,05)$ do grupo I apresentando concentração média de 40,46 $\pm 18,13 \times 10^{9}$ sptz/mL em relação à variação mensal. A concentração espermática mínima observada ocorreu em novembro de $2007\left(18,12 \times 10^{9}\right.$ sptz/mL) e a máxima em dezembro de 2007 $\left(73,42 \times 10^{9} \mathrm{sptz} / \mathrm{mL}\right)(\mathrm{p}<0,5)$, coincidindo com baixo volume seminal. A produção total de espermatozoides do grupo I foi de 115,79 x $10^{9} \mathrm{sptz} /$ indivíduo e do grupo NI foi de $22,25 \times 10^{9}$ sptz/indivíduo.

O volume seminal médio foi de 5,05 $\pm 2,08$ $\mathrm{mL}$ no grupo I e $0,55 \pm 0,52 \mathrm{~mL}$ no grupo NI. O maior volume de sêmen observado para o grupo I ocorreu em novembro de 2007, assim como a menor concentração espermática; entretanto, foi também observada no mês de janeiro de 2008, mostrando uma grande variação no volume seminal neste grupo durante aos meses do ano. Viveiros e Godinho(2009) mencionam o volume seminal como um fator importante no processo reprodutivo e que tanto em espécies migratórias na época de reprodução quanto em animais induzidos com hormônio é bastante variável, e a concentração espermática varia de 10,9 a $69,9 \times 10^{9} \mathrm{sptz} / \mathrm{mL},(L$. obtusidens e $R$. quelen), respectivamente. Os valores médios de $\mathrm{pH}$ foram de $8,17 \pm 0,38$ para o grupo I e $8,06 \pm 0,22$ para o grupo NI; e osmolaridade de $319 \pm 13$ a $313 \pm 20 \mathrm{mOsml} /$ $\mathrm{kg}$ para nos grupos I e NI, respectivamente.

Os resultados deste trabalho mostram

Tabela I. Média e desvio padrão dos parâmetros seminais do tambaqui C. macropomum nos grupos induzido (I) e não induzido (NI) hormonalmente com $2 \mathrm{mg} / \mathrm{ECP} / \mathrm{kg} / \mathrm{PV}$, Pentecoste, Ceará, Brasil. (Mean and standard desviation of tambaqui C. macropomum seminal parameters into hormonally induced (I) and no-induced (NI) groups with $2 \mathrm{mg} / \mathrm{ECP} / \mathrm{kg} / \mathrm{AW}$, Pentecoste, Ceará, Brazil).

\begin{tabular}{lcc}
\hline Parâmetros & Induzidos & Não induzidos \\
\hline Volume $(\mathrm{mL})$ & $5,05 \pm 2,00^{\mathrm{a}}$ & $0,55 \pm 0,50^{\mathrm{b}}$ \\
$\mathrm{pH}$ & $8,21 \pm 0,39^{\mathrm{a}}$ & $8,09 \pm 026^{\mathrm{a}}$ \\
Osmolaridade $(\mathrm{mOsm} / \mathrm{kg})$ & $320,51 \pm 14,58^{\mathrm{a}}$ & $313,40 \pm 27,20^{\mathrm{a}}$ \\
Concentração $\left(\times 10^{9} \mathrm{sptz} / \mathrm{mL}\right)$ & $22,93 \pm 9,79^{\mathrm{b}}$ & $40,46 \pm 18,31^{\mathrm{a}}$ \\
Concentração total sptz/mês $\left(\times 10^{9} \mathrm{sptz} / \mathrm{mL}\right)$ & $115,79 \times 10^{9 \mathrm{a}}$ & $22,25 \times 10^{9 \mathrm{~b}}$ \\
\hline
\end{tabular}

Letras diferentes entre colunas $(p<0,05)$.

Archivos de zootecnia vol. 60, núm. 232, p. 1266. 
que houve variação entre os meses do ano durante o estudo, no entanto, tal fato não inviabiliza a utilização do sêmen desta espécie durante todo o ano, especialmente sob indução hormonal. Esta variação foi especialmente observada no parâmetro volume seminal, tendo reflexo direto sobre a concentração espermática.

Segundo Taitson e Godinho (2003) há uma grande variação nos dados de concentração espermática em peixes em geral, parecendo ser uma influência espécie-específica, havendo também grandes diferenças individuais, acarretando informações diferenciadas dentro da mesma espécie. Observa-se, para peixes de água doce, uma

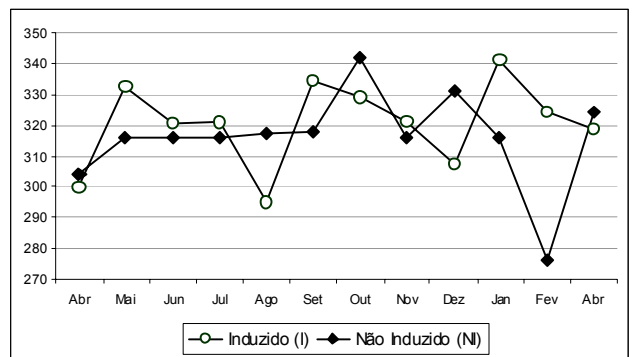

(A) Osmolaridade $(\mathrm{mOsm} / \mathrm{kg}) ; \mathrm{p}>0,05$.

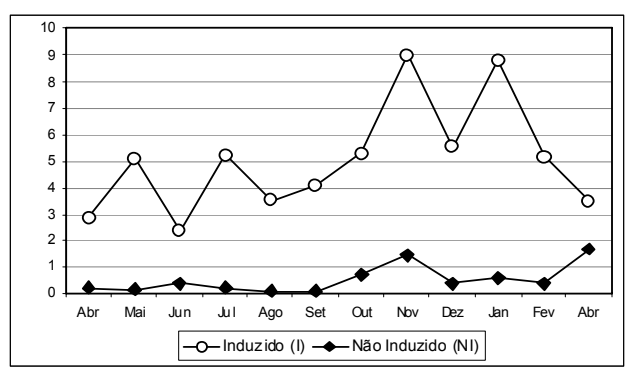

(C) Volume seminal (mL); $\mathrm{p}<0,05$. variação de $1,33 \mathrm{~mL}$ (com Leporinus elongatus; Taitson e Godinho, 2003 ) e 1,0 mL (Viveiros e Godinho, 2009) a 14,5 mL em Brycon orbignyanus, (Viveiros e Godinho, 2009) no volume seminal coletado inclusive com indução hormonal (Ciereszko e Dabrowskib, 1993; Andrade-Talmelli et al., 2001; Taitson e Godinho, 2003; Ákos et al., 2003; Fresneda et al., 2004; Bombardelli et al., 2006; Viveiros et al., 2008). Menezes et al. (2008) obtiveram concentrações espermáticas de tambaqui C. macropomum de 35 x $10^{9} \mathrm{sptz} / \mathrm{mL}$ após indução com $1 \mathrm{mg} / \mathrm{kg}$ de solução com hipófise de carpa C. carpio.

Farias et al. (1999) estudando o volume seminal de tambaqui C. macropomum,

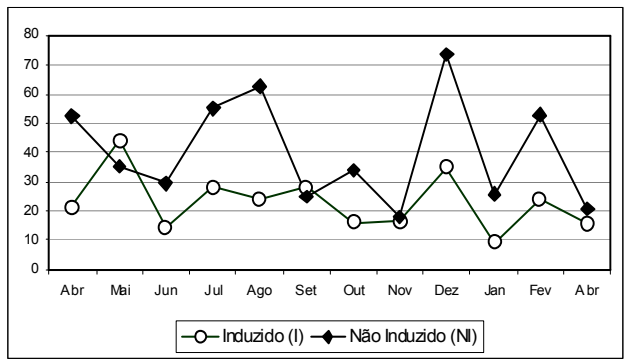

(B) Concentração espermática (x10 $\mathrm{sptz} / \mathrm{mL}) ; \mathrm{p}<0,05$.

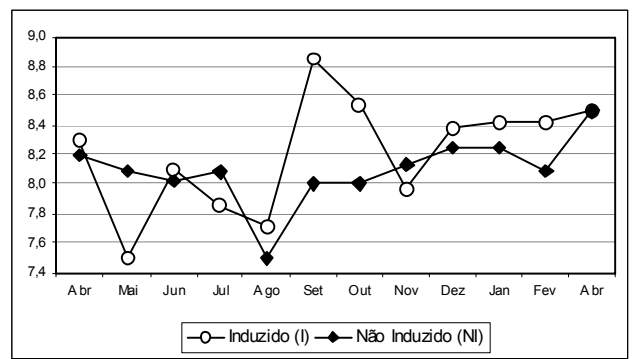

(D) $\mathrm{pH}(0-14) ; \mathrm{p}>0,05$.

Figura 1. Parâmetros seminais do tambaqui C. macropomum nos grupos induzido (I) e não induzido (NI) hormonalmente com $2 \mathrm{mg} / E C P / \mathrm{kg} / P V$ no período de abril de 2007 a abril de 2008, em Pentecoste, Ceará, Brasil. Quanto a: (A) Osmolaridade, (B) Concentração espermática, (C) Volume seminal e (D) pH. (Tambaqui C. macropomum seminal parameters into hormonally induced (I) and no-induced (NI) groups with $2 \mathrm{mg} / \mathrm{ECP} / \mathrm{kg} / \mathrm{AW}$ during the period between April 2007 to April 2008, at Pentecoste, Ceará, Brazil. Related to: (A) Osmolarity, (B) Spermatic concentration, (C) Seminal volume, and (D) pH). 
induzidos com GnRH (100 $\mu \mathrm{g} / \mathrm{kg} / \mathrm{PV})$, encontraram volume seminal de $5,0 \pm 1,2 \mathrm{~mL}$, resultado semelhante ao do presente estudo no grupo I de tambaqui utilizando $2 \mathrm{mg} /$ $\mathrm{ECP} / \mathrm{kg} / \mathrm{PV}$ que obteve média de 5,05 $\pm 2,00$ $\mathrm{mL}$. Entretanto, os resultados destes autores, quando se compara os dados em peixes não induzidos hormonalmente, observamse valores médios superiores de volume $\operatorname{seminal}(2,0 \pm 0,8 \mathrm{~mL} v s .0,55 \pm 0,5 \mathrm{~mL})$.

Variação da concentração espermática também é observada entre as espécies, encontrando-se relatos de concentração mínima, em peixe de água doce, de $1,97 \times 10^{9} \mathrm{sptz} /$ $\mathrm{mL}$ (Rhamdia quelen; Bombardelli et al., 2006) e máxima de $41,58 \times 10^{\circ} \mathrm{sptz} / \mathrm{mL}$ (Perca jlavescens; Ciereszko e Dabrowski, 1993). Farias et al. (1999) e Menezes et al. (2008) obtiveram com tambaqui concentrações espermáticas da ordem de $35 \times 10^{9} \mathrm{sptz} / \mathrm{mL}$ e Folgli da Silveira et al. (1990) citado por Viveiros e Godinho (2009) obtiveram concentrações de $23,3 \times 10^{9} \mathrm{sptz} / \mathrm{mL}$ em $P$. mesopotamicus. Encontrou-se variação média mensal de 9,12 a $73,42 \times 10^{9} \mathrm{sptz} / \mathrm{mL}$, demonstrando que há diferenças significativas na concentração não só entre espécies, mas também individuais, dentro da mesma espécie. Observou-se ainda que com a indução hormonal ocorre diminuição da concentração espermática $\left(22,93 \times 10^{9} v s\right.$. $40,46 \times 10^{9} \mathrm{sptz} / \mathrm{mL}$ nos grupos I e NI), porém há um aumento significativo no volume seminal coletado $(5,05$ vs. $0,55 \mathrm{~mL}$ nos grupos I e NI, respectivamente) o que contribui positivamente para o manuseio do sêmen em reprodução assistida. Vale ressaltar que os valores encontrados neste estudo são inferiores aos de Menezes (2003) que também trabalharam com tambaqui (induzido com ECP $1 \mathrm{mg} / \mathrm{kg} / \mathrm{PV}$ ) onde encontram concentração espermática média de $35 \times 10^{9} \mathrm{sptz} / \mathrm{mL}$ e volume variando de 6 a $12 \mathrm{~mL}$.

$\mathrm{O} \mathrm{pH}$ do sêmen de peixes água doce varia de 6,5 a 8,5, segundo Tabares et al. (2005), portanto, os dados encontrados para tambaqui $(8,21 \pm 0,3$ e 8,09 $\pm 0,26$ nos grupos I e NI) estão de acordo com esta assertiva.
De acordo com Morisawa e Susuki (1980) a osmolaridade do sêmen de peixes apresenta-se em torno de $300 \mathrm{mOsm} / \mathrm{kg}$, corroborando com os dados encontrados neste estudo para tambaqui (276 a $342 \mathrm{mOsm} /$ $\mathrm{kg})$. A variabilidade apresentada neste parâmetro parece ter relação com características individual e espécie-específicas, pois as mesmas também foram encontradas em trabalhos com ciprinídeos (254 a 346 mOsm/ $\mathrm{kg}$; Alavi e Cosson, 2006) e salmonídeos (232 a 322 mOsm/kg; Alavi e Cosson, 2006). No presente estudo foi observada osmolaridade média para tambaqui induzido hormonalmente $(2 \mathrm{mg} / \mathrm{ECP} / \mathrm{kg} / \mathrm{PV})$ de $320,51 \mathrm{mOsm} /$ $\mathrm{kg}$, superior ao encontrado por Farias et al. (1999) com tambaqui (induzido com GnRH: $100 \mu \mathrm{g} / \mathrm{kg} / \mathrm{PV}$ ) que foi de $274 \mathrm{mOsm} / \mathrm{kg}$.

O conhecimento da osmolaridade média do sêmen de peixe teleósteos, como no caso o tambaqui, é de extrema importância na reprodução assistida, uma vez que seus espermatozóides permanecem quiescentes na osmolaridade do plasma seminal, fator crítico aos espermatozoides que, após a ativação, tem uma viabilidade média de aproximadamente 2 minutos. Dentre os fatores que ocasionam a ativação da motilidade espermática está o choque osmótico que os mesmos sofrem ao encontrarem na água doce (meio hipotônico; $4 \mathrm{mOsm} / \mathrm{kg}$ ) ou salgada (meio hipertônico, $1000 \mathrm{mOsm} / \mathrm{kg}$ ), no caso de teleósteos marinhos (Alavi e Cosson, 2006).

\section{CONCLUSÕES}

A indução hormonal é necessária para garantir a quantidade de parâmetros seminais para o processo de reprodução do tambaqui C. macropomum Cuvier, 1818. A concentração espermática entre os animais induzidos é menor que as dos não induzidos, porém, a diferença não compromete o processo reprodutivo, pois o volume compensa a diferença de concentração. Foi comprovado que o intervalo de dois meses é suficiente para permitir o repouso 


\section{SÊMEN DE TAMBAQUI (COLOSSOMA MACROPOMUM) EM LATITUDE EQUATORIAL}

necessário entre as induções hormonais. Osmolaridade e $\mathrm{pH}$ nos dois grupos, induzidos e não induzidos, mantêm-se nos limites aceitáveis para as espécies de água doce em condições edafoclimáticas equatoriais de Pentecoste-Ceará durante todo o ano.

\section{AGRADECIMENTOS}

Ao Programa de Pós-Graduação em

\section{BIBLIOGRAFIA}

Alavi, S.M.H. and Cosson, J. 2006. Sperm motility in fishes. (II) Effects of ions and osmolality: A review. Cell Biol. Int., 30: 1-14.

Ákos, H., Edit, M. and Béla, U. 2003. Cryopreservation of common carp sperm. Aquat. Living Resour., 16: 457-460.

Andrade-Talmelli, E.F., Kavamoto, E.T. e FenerichVerani, N. 2001. Características seminais da piabinha, Brycon insignis (Steindachner, 1876), após estimulação hormonal. B. Inst. Pesca, 27 149-154.

Bombardelli, R.A., Mörschbächer, E.F., Campagnolo, R., Sanches, E.A. e Syperreck, M.A. 2006. Dose inseminante para fertilização artificial de ovócitos de jundiá cinza, Rhamdia quelen (Quoy e Gaimardm, 1824), Rev. Bras. Zootecn., 5: 1251-1257.

Ciereszko, A. and Dabrowski, K. 1993. Estimation of sperm concentration of rainbow trout whitefish and yellow perch using a spectrophotometric technique. Aquaculture, 109: 367-373.

Cruz-Casallas, P.E., Lombo-Rodríguez, D.A. and Velasco-Santamaría, Y.M. 2005. Milt quality and spermatozoa morphology of captive Brycon siebenthalae (Eigenmann) broodstock. Aquacult. Res., 36: 682-686.

Farias, J.O., Nunes, J.F., Carvalho, M.A.M. e Salgueiro, C.C.M. 1999. Avaliação in vitro e in vivo do sêmen de tambaqui (Colossoma macropomum) conservado a temperatura ambiente e criopreservado em água de coco. Rev. Cient. Prod. Animal., 1: 44-58.

Fresneda, A., Lenis, G., Agudelo, E. e Angel, M.O. 2004. Espermiación inducida y crioconservación de semen de cachama blanca (Piaractus brachypomus). Rev. Col. Cienc. Pec., 17 (supp): 46-52.
Ciências Veterinárias da Faculdade de Veterinária da Universidade Estadual do Ceará; ao Centro de Pesquisas em Aquicultura do DNOCS em Pentecoste-CE em nome do Msc. Pedro Eymard Pinto Mesquita e da Diretora Geral Dra. Renata Pollari Telles Borrigueiro, pelo apoio na execução do trabalho experimental e à FUNCAP pela bolsa de doutorado concedida.

FUNCEME. 2008. Relatório de Pluviometria por faixa de anos. Estado do Ceara, 1974-2008, anos 2007 e 2008. Município: Pentecoste, Posto: Pentecoste, Micro-região: 11, Código: 115, Resumo de chuvas nos dados fornecidos pela Funceme.

Kavamoto, E.T. e Fogli da Silveira, W. 1986. Características físicas, químicas e microscópicas do sêmen de bagre (Rhamdia hilarii valenciennes, 1840) em condições de campo. B. Inst. Pesca, 13: $95-100$.

Menezes, J.T.B. 2003. Programa banco de sêmen de tambaqui silvestre Colossoma macropomum, Cuvier 1818. Rev. Panor. Aqüicult., 3: 76.

Menezes, J.T.B., Queiroz, L.J., Costa, D.C.R. e Menezes, J.R.J.B. 2008. Avaliação espermática pós-descongelamento em tambaqui, Colossoma macropomum (Cuvier, 1818). Acta Amazonica, 38: 365-368.

Morisawa, M. and Suzuki, K. 1980. Osmolality and potassium ion: their roles in initiation of sperm motility in teleosts. Science, 210: 1145-1147.

Muniz, J.A.S.M. 2006. Influência do LHRH comum na ovulação induzida do tambaqui Colossoma macropomum (Cuvier) (Characiforme, Characidae), em diferentes fotoperíodos. Dissertação de Mestrado. Programa de Pós-Graduação em Recursos Pesqueiros e Aqüicultura. Universidade Federal Rural de Pernambuco. Recife. 48 pp. Ponzi Junior, M. 2003. Otimização da taxa de fertilização e eclosão de larvas de tambaqui, Colossoma macropomum (Cuvier, 1816) sem instrumentos. Dissertação de Mestrado. Programa de Pós-Graduação em Recursos Pesqueiros e Aqüicultura. Universidade Federal Rural de Pernambuco. Recife. 23 pp.

Salgueiro, C.C.M. e Nunes, J.F. 1999. Estudo de 
VIEIRA, CARVALHO, SALMITO-VANDERLEY, SALGUEIRO, VIVEIROS, MOURAENUNES

características testiculares e espermáticas de caprinos e ovinos. Rev. Bras. Reprod. Anim. 23: 231-232.

Shimoda, E., Andrade, D.R., Cruz, G.M., Silva, J.F.S. e Godinho, H.P. 1999. Caracterização química do plasma seminal do pacu (Piaractus mesopotâmicus; Holmberg, 1887) hipofisado. Rev. Bras. Reprod. Anim., 23: 151-478.

Shimoda, E. 2004. Análise e criopreservação do sêmen da piabanha, Brycon insignis (Steindachner, 1877) (Pisces, Characidae). Tese de Doutorado. Universidade Estadual do Norte Fluminense Darcy Ribeiro. Campos dos Goytacazes. Rio de Janeiro. 124 pp.

Silva, A., Carneiro, S. e Melo, F.R. 1981. Desova induzida de tambaqui, Colossoma macropomum, Cuvier, 1818, com de hipófise de curimatã comum, Prochilodus cearensis Steindachner. Coletânea de Trabalhos Técnicos. DNOCS. Fortaleza (CE).

Silva, J.W.B., Nobre, M.I.S., Pinheiro, F.A. e Sobrinho, A.C. 1984. Resultado de um experimento de policultivo de tambaqui Colossoma macropomum, Cuvier, 1818, hibrido de tilápia, (Oreochromis hornorum TREW x O. niloticus L., 1766) e carpa espelho Cyprinus carpio L., 1758 vs. specularis. Bol. Téc. do DNOCS, 42: 63-89.
Silva, J.A.M., Pereira-Filho, M. e Oliveira-Pereira, M.I. 1999. Digestibility of seeds consumed by tambaqui (Colossoma macropomum Cuvier, 1818): an experimental approach. In: Val, A.L., Almeida-Val, V.M. (Org.). Biology of Tropical Fishes. INPA. Manaus. $460 \mathrm{pp}$.

Tabares, J., Tarazona, A. e Oliveira, M. 2005. Fisiología de la activación del espermatozoide en peces de agua dulce. Rev. Coml. Cienc. Pec., 18: 149-160.

Taitson, P.F. and Godinho, H.P. 2003. Evaluation of fish sperm concentration using two counting chambers. Arq. Bras. Med. Vet. Zoo., 55: 238239.

Villacorta-Correa, M.A. and Saint-Paul, U. 1999. Structural indexes and sexual maturity of tambaqui Colossoma macropomum (Cuvier, 1818) (Characiformes: Characidae) in Central Amazon, Brazil. Rev. Bras. Biol., 59: 637-652. Viveiros, A.T.M., Orfão, L.H., Maria, A.N. and Allaman I.B. 2008. A simple, inexpensive and successful freezing method for curimba Prochilodus lineatus (Characiformes) semen. Anim. Reprod. Sci., 112: 293-300.

Viveiros, A.T.M. and Godinho, H.P. 2009. Sperm quality and cryopreservation of Brazilian freshwater fish species: a review. Fish Physiol. Biochem., 35: 137-150. 